Supplement of Hydrol. Earth Syst. Sci. Discuss., 12, 13301-13358, 2015

http://www.hydrol-earth-syst-sci-discuss.net/12/13301/2015/

doi:10.5194/hessd-12-13301-2015-supplement

(C) Author(s) 2015. CC Attribution 3.0 License.

(c) (i)

Supplement of

\title{
The importance of topography controlled sub-grid process heterogeneity in distributed hydrological models
}

\author{
R. C. Nijzink et al. \\ Correspondence to: R. C. Nijzink (r.c.nijzink@ @udelft.nl)
}

The copyright of individual parts of the supplement might differ from the CC-BY 3.0 licence. 


\section{Ranked Probability Score}

2 The Ranked Probability Score ( $\mathrm{S}_{\mathrm{RP}}$; Wilks, 2005) was adapted as a measure for the magnitude

3 of the expected model improvement or deterioration. Originally, $\mathrm{S}_{\mathrm{RP}}$ was designed to estimate

4 the "distance" between an observation and an empirical cumulative distribution function,

5 based on the area enclosed between the two (Figure 6). The Ranked Probability Score is given 6 by:

$7 \quad S_{R P}=\frac{1}{M-1} \sum_{m=1}^{M}\left[\left(\sum_{k=1}^{m} p_{k}\right)-\left(\sum_{k=1}^{m} o_{k}\right)\right]^{2}$,

8 where $\mathrm{M}$ is the length of the distribution of performances of a certain signature, $\mathrm{p}_{\mathrm{k}}$ the 9 probability of a certain signature performance to occur and $\mathrm{o}_{\mathrm{k}}$ the probability of the observation to occur. In our case $o_{k}$ is a step function, which is either 1 or 0 . For example, $E_{N S}$

11 has it's optimal value at 1 . Thus, as there is only one time series, it has a step distribution

12 function at 1 . The model runs will have a cumulative distribution function, as multiple sets of parameters are considered as feasible. This distribution function will be close to 1 in case of model with a relatively good performance. The difference of the $S_{\mathrm{RP}}$ between two models was used here as a measure to identify and quantify improvement.

16

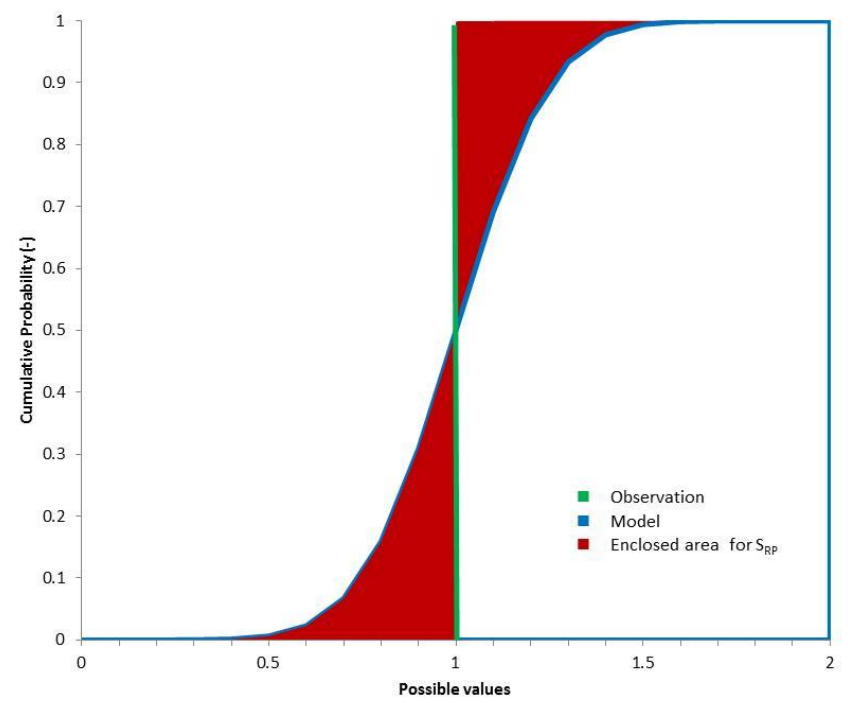

17 Figure $\mathrm{S} 1$. Graphical illustration of the ranked probability score $\mathrm{S}_{\mathrm{RP}}$. The enclosed area (red) between model (blue) and observation (green) determines the score. 


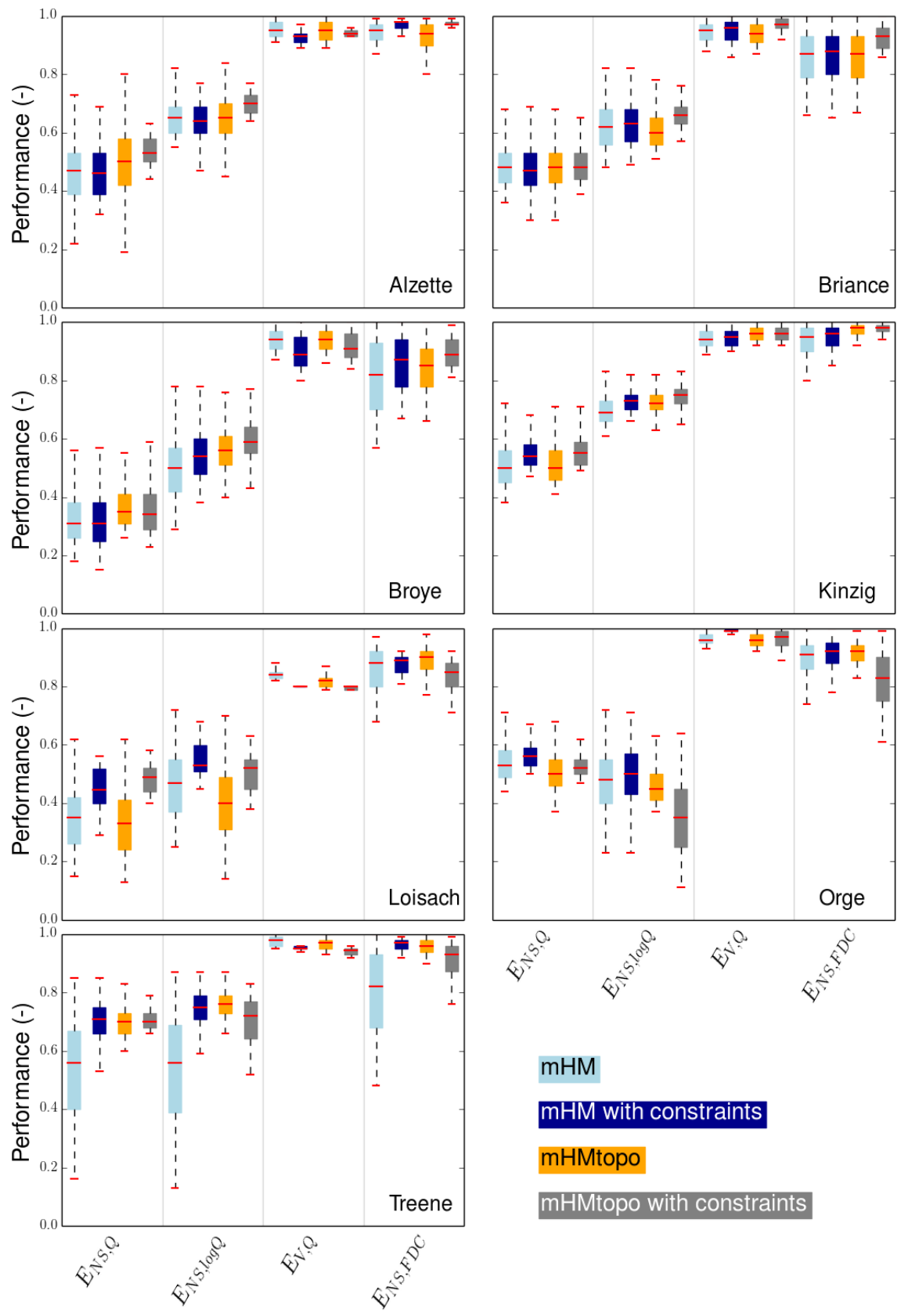

2 Figure S2. Nash-Sutcliffe efficiency $\left(\mathrm{E}_{\mathrm{NS}, \mathrm{Q}}\right)$, log Nash-Sutcliffe efficiency $\left(\mathrm{E}_{\mathrm{NS}, \log \mathrm{Q}}\right)$, volume 3 error $\left(\mathrm{E}_{\mathrm{V}, \mathrm{Q}}\right)$ and $\log$ Nash-Sutcliffe efficiency of the flow duration curve $\left(\mathrm{E}_{\mathrm{NS}, \mathrm{FDC}}\right)$ for the 4 seven catchments in the calibration periods. The optimal value for all four criteria is 1 , 5 whereas 0 is regarded to have a low performance. The boxplots are formed by the Pareto 6 space spanned by the four objective functions. 

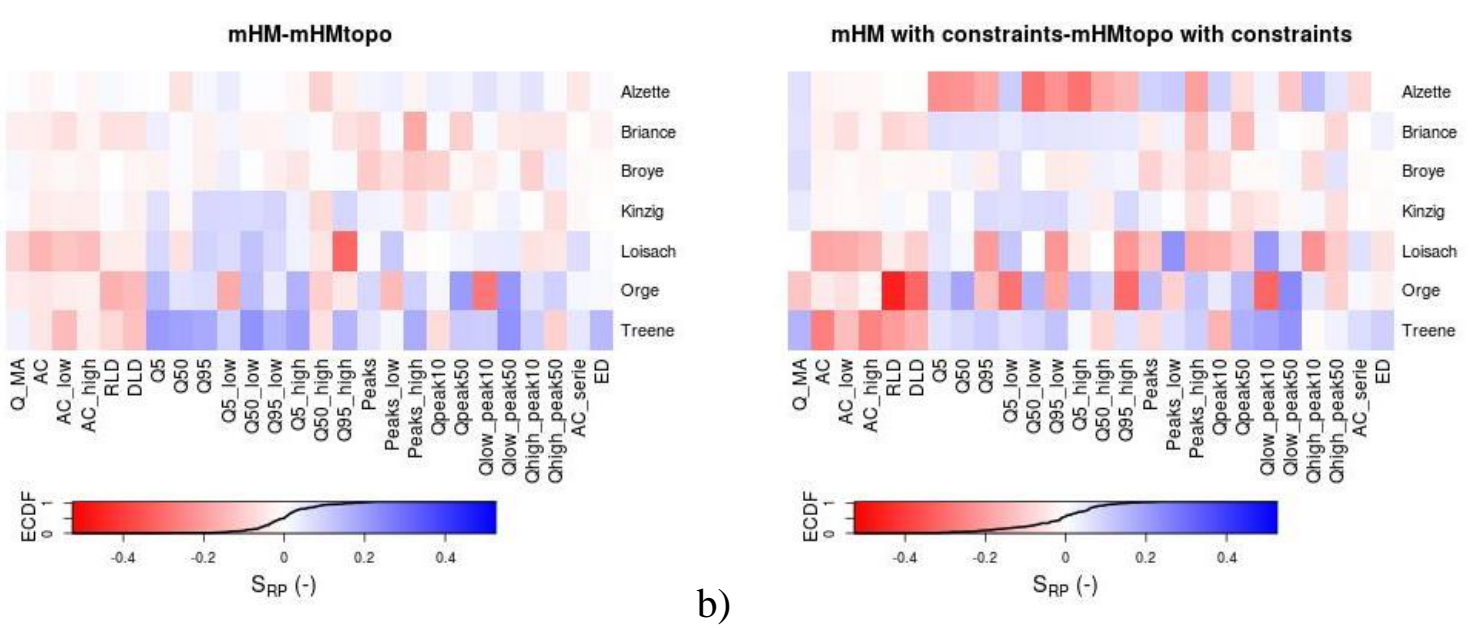

a)
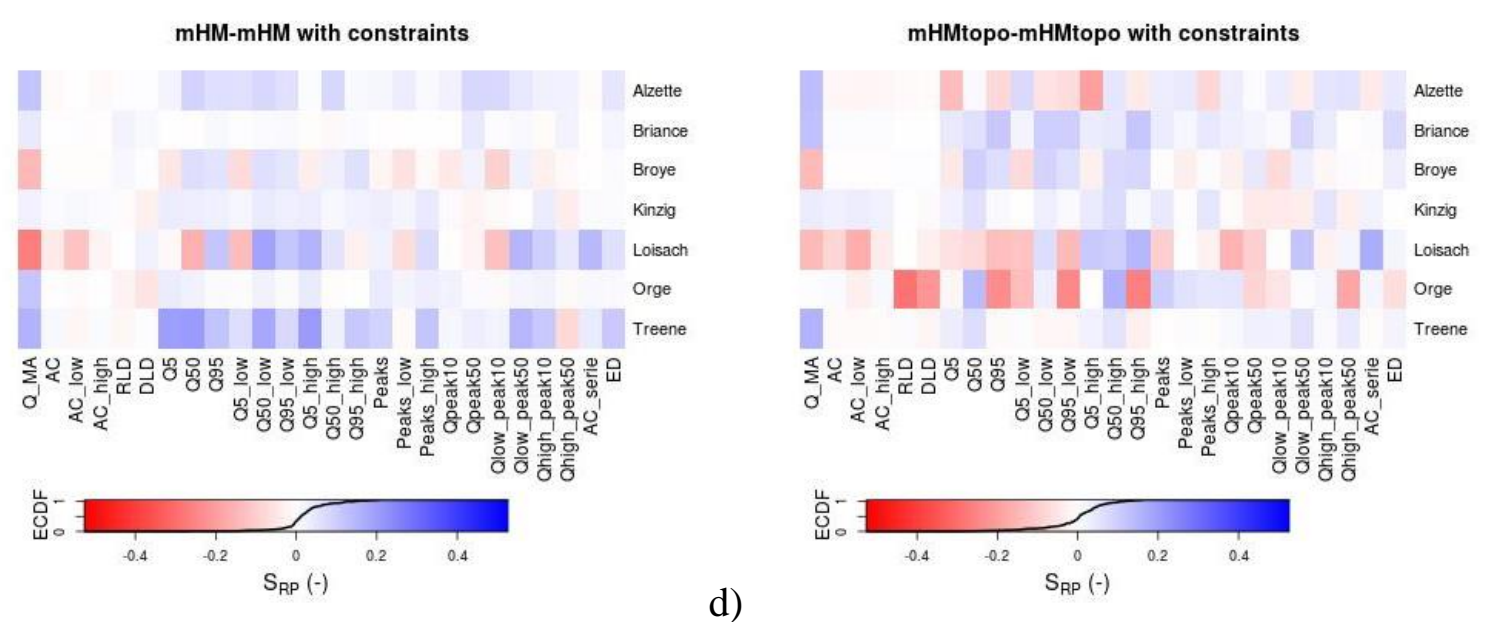

$2 \mathrm{c})$

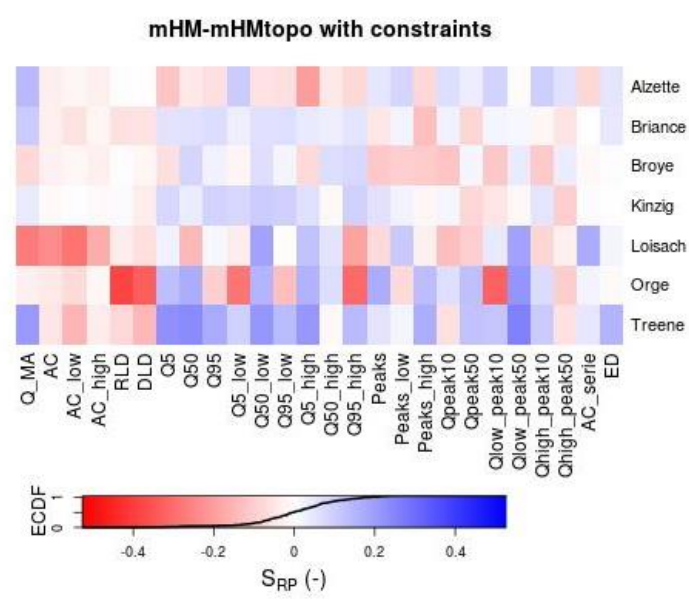

3 e)

d)

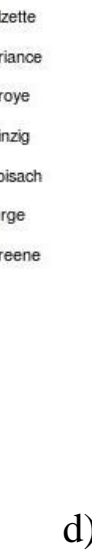

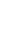

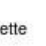

Briance

Loisach

Treene

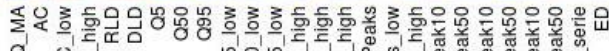

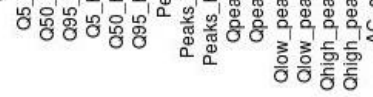

e)

4 Figure S3. Difference in Ranked Probability Scores between (a) mHM and mHMtopo without

5 constraints and (b) with constraints, (c) mHM with and without constraints, (d) mHMtopo

6 with and without constraints (e) the base case mHM with the constrained mHMtopo case. The

7 colours are linearly related to scores between the most negative values (darkred), 0 (white)

8 and the most positive values (darkblue), where positive values indicate an improvement. An 
1 empirical cumulative distribution function based on all values has been added to assess the 2 distribution of occurring score differences.

3 

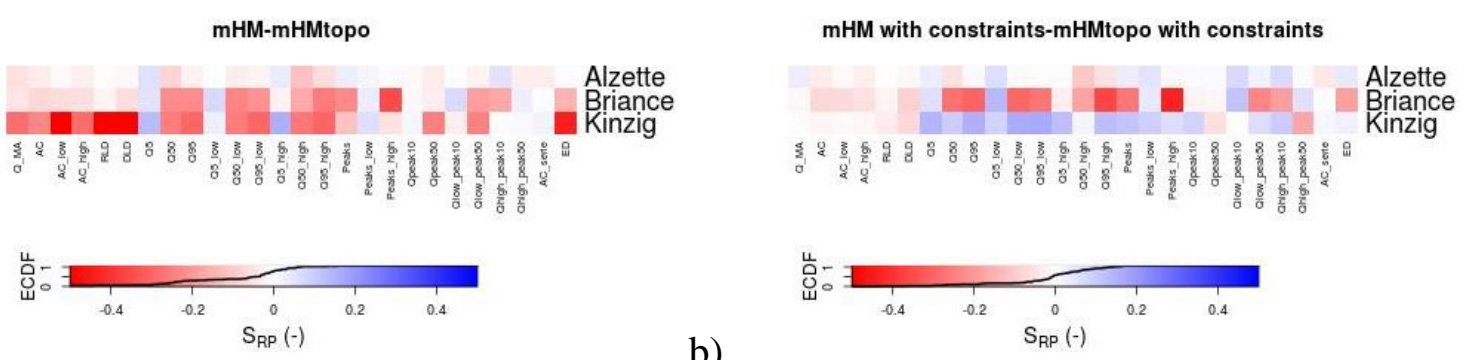

a)

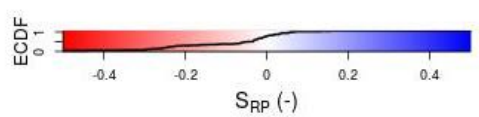

2

c)

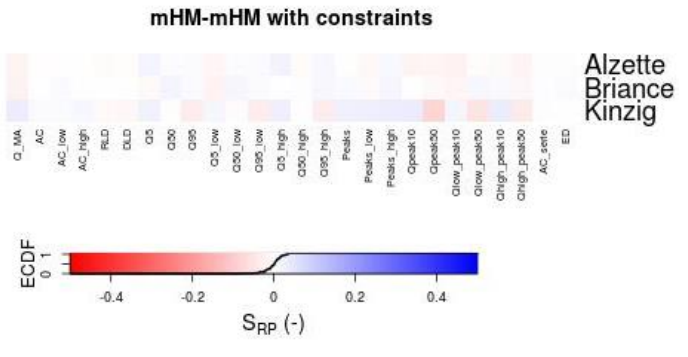

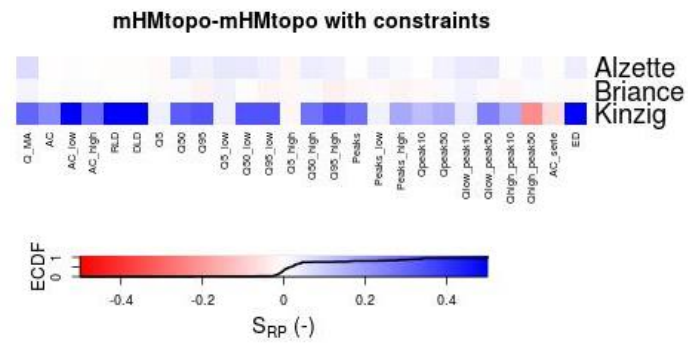

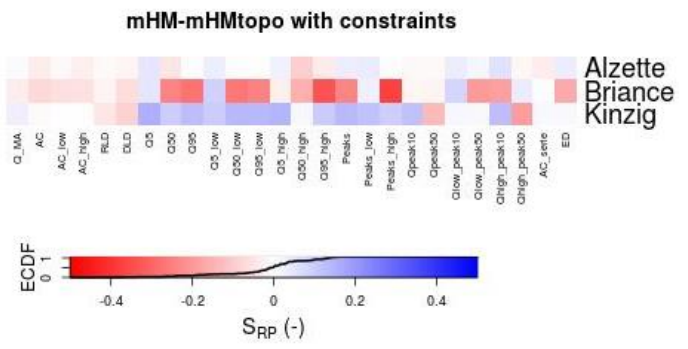

e)

4 Figure S4. Difference in Ranked Probability Scores between (a) mHM and mHMtopo without 5 constraints and (b) with constraints, (c) mHM with and without constraints, (d) mHMtopo 6 with and without constraints and (e) the base case mHM with the constrained mHMtopo after 7 the transfer of global parameters. The colours are linearly related to scores between the most 8 negative values (darkred), 0 (white) and the most positive values (darkblue), where positive 9 values indicate an improvement. An empirical cumulative distribution function based on all values has been added to assess the distribution of occurring score differences. 